\title{
Does the data do the talking? Confirmation bias in the interpretation of data visualizations
}

\author{
Itai Yanai ${ }^{1, *}$ and Martin Lercher ${ }^{2, *}$ \\ ${ }^{1}$ Institute for Computational Medicine, NYU Langone Health, New York, NY 10016, USA, \\ 2 Institute for Computer Science \& Department of Biology, Heinrich Heine University, 40225 \\ Düsseldorf, Germany \\ *Corresponding authors: itai.yanai@nyulangone.org, martin.lercher@hhu.de
}

\section{Abstract}

When analyzing the results of an experiment, we often assume that the interpretation of the data is a straightforward act; however, experiments on reproducibility that have large numbers of scientists analyze the same dataset show that even subtle differences in workflows can lead to drastically different results. Beyond the use of different analysis methods, one potential reason for different conclusions from the same data is confirmation bias, a phenomenon well documented in psychology. We reasoned that confirmation bias might not only influence the choice of analysis methods and depth of analysis, but that it might even shape how different researchers interpret the same graphical representation of data. To test this notion, we designed a simple experiment, in which we contrast the prior expectation of participants' for the relationship between two variables (income and happiness) with their interpretation of a corresponding data plot. We artificially engineered the data such that under superficial examination, it shows an overall negative correlation, while a closer look at distinct age groups (distinguished by color) reveals a within-group positive correlation. Prior to showing the plot, we had asked the participants whether they expected a positive or a negative relationship. We found that participants who expected a positive correlation were more than twice as likely to detect the positive within-age group correlation than those expecting a negative correlation. This simple experiment demonstrates the presence of confirmation bias in the interpretation of graphical data representations. 


\section{Introduction}

While in scientific research, the prevailing notion is that 'the data speaks for itself', recent work has begun to challenge this notion. In one study, 70 independent teams were asked to analyze functional magnetic resonance images and test 9 specific hypotheses [1]. Strikingly, no two teams - all but one of which were experts in this type of analysis - chose identical work flows to analyze the dataset, and sets of teams reported contradictory effects based on the same dataset. In another study, 73 teams used the same data to test a single hypothesis; the "tremendous variation" in conclusions led the researchers to conclude a "vast universe of research design variability normally hidden from view" [2]. These and other studies [3-8] demonstrate that data is not transparent but requires interpretation. Yet the biases that impinge upon the interpretation are not well understood.

One important reason for differences in conclusions from the same data cited in previous studies on reproducibility is that different scientists used different workflows. However, it is conceivable that pre-existing biases on the level of the working group or the individual scientist influenced the conclusions. Psychologists use the term 'confirmation bias' to denote the propensity of an individual to view new evidence as supporting one's beliefs [9, 10]. For example, the effects of confirmation bias on scientific peer review - where reviewers tend to judge manuscripts much more harshly if their conclusions contradict the reviewer's assumptions - are well documented [11-13].

We sought to design an experiment that would efficiently and elegantly test the degree to which pre-existing biases shape how analysts see data. The experiments cited above studied the analysis workflows of the experiments, and thus the observed differences in interpretation could be attributed to decisions made along the way, each leading to a different set of graphs and statistics. We wondered if different conclusions would also be made in an even more extreme setting: would participants interpret an identical plot of a dataset differently? And second, would we be able to correlate the interpretation of a plot with the bias of the participants?

\section{Results}

To test this, we asked each of 171 undergraduate university students of computer science at Heinrich Heine University Düsseldorf what general correlation they expected between wealth and happiness: positive or negative. With these biases established, we then showed all participants the same plot of the relationship between the wealth and happiness of 1000 individuals in a fictitious dataset (Figure 1). We asked each participant to answer 'yes' or 'no' to the following question: "Does the data suggest that wealth leads to happiness?". We hypothesized that participants would be more likely to report an observation that agreed with their stated expectation than one that would contradict it.

Collectively, the data points in Figure 1 (indicating individuals) showed a negative correlation. A positive correlation could be seen, however, among the data points corresponding to each age 
group, distinguished by four colors in the Figure. The figure thus is an instance of Simpson's Paradox, where the correlation between two variables changes sign after controlling for another variable [14]. Here, age is apparently a confounding factor. Not controlling for this factor results in an overall negative correlation; only when analyzing age groups separately does a positive correlation emerge. The notion of Simpson's Paradox is not obvious and needs to be learned, and so we did not expect all participants to identify that the data supports a positive relationship between wealth and happiness.

\section{Does the data suggest that wealth leads to happiness?}

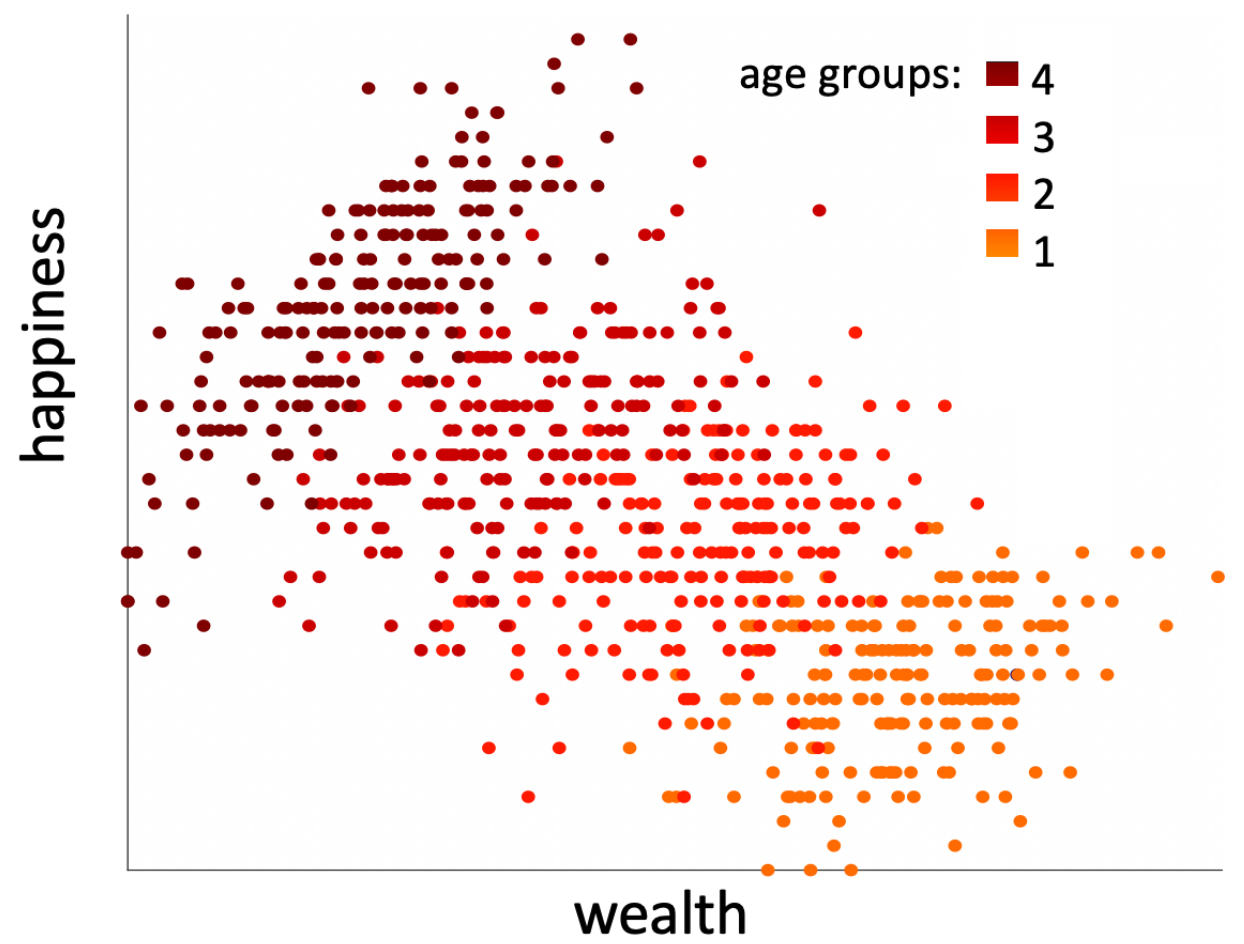

Figure 1. The artificial dataset on happiness versus income shown to the participants. Each dot represents one individual; colors denote age groups (1=youngest, 4=oldest).

\section{The data tells you what you expect}

In a first replicate of the experiment (Dataset 1), we questioned $N=76$ first semester students. Table 1A shows the corresponding contingency table, contrasting the data-inferred causality (coded as negative or positive observed relationships, columns) with the correlations expected by the students before being confronted with the data (negative or positive, rows). The odds ratio calculated for the data in Table 1A is 3.07; a one-sided Fisher's exact test shows that this is marginally statistically significant at the $5 \%$ level, $P=0.049$. Students were 2.3 times more likely to observe a positive correlation if they expected to see one $(19 / 51=0.373$ vs. $4 / 25=0.160)$. 
Table 1. Contingency tables for the expected versus observed correlations.
(A) A summary of Dataset 1 (Suppl. Table S1).
(B) A summary of Dataset 2 (Suppl. Table S2).
(C) Combined analysis of Datasets 1 and 2.

\begin{tabular}{|c|c|c|c|c|c|c|c|c|c|c|c|}
\hline A & $\begin{array}{l}\text { neg. } \\
\text { obs. } \\
\text { rel. }\end{array}$ & $\begin{array}{l}\text { pos. } \\
\text { obs. } \\
\text { rel. }\end{array}$ & $\begin{array}{l}\text { row } \\
\text { sums }\end{array}$ & B & $\begin{array}{c}\text { neg. } \\
\text { obs. } \\
\text { rel. }\end{array}$ & $\begin{array}{l}\text { pos. } \\
\text { obs. } \\
\text { rel. }\end{array}$ & $\begin{array}{l}\text { row } \\
\text { sums }\end{array}$ & C & $\begin{array}{c}\text { neg. } \\
\text { obs. } \\
\text { rel. }\end{array}$ & $\begin{array}{c}\text { pos. } \\
\text { obs. } \\
\text { rel. }\end{array}$ & $\begin{array}{l}\text { row } \\
\text { sums }\end{array}$ \\
\hline $\begin{array}{l}\text { neg. } \\
\text { corr. } \\
\text { expct. }\end{array}$ & 21 & 4 & $\begin{array}{c}25 \\
(33 \%)\end{array}$ & $\begin{array}{l}\text { neg. } \\
\text { corr. } \\
\text { expct. }\end{array}$ & 24 & 3 & $\begin{array}{c}27 \\
(28 \%)\end{array}$ & $\begin{array}{l}\text { neg. } \\
\text { corr. } \\
\text { expct. }\end{array}$ & 45 & 7 & $\begin{array}{c}52 \\
(30 \%)\end{array}$ \\
\hline $\begin{array}{l}\text { pos. } \\
\text { corr. } \\
\text { expct. }\end{array}$ & 32 & 19 & $\begin{array}{c}51 \\
(67 \%)\end{array}$ & $\begin{array}{l}\text { pos. } \\
\text { corr. } \\
\text { expct. }\end{array}$ & 45 & 23 & $\begin{array}{c}68 \\
(72 \%)\end{array}$ & $\begin{array}{l}\text { pos. } \\
\text { corr. } \\
\text { expct. }\end{array}$ & 77 & 42 & $\begin{array}{c}119 \\
(70 \%)\end{array}$ \\
\hline $\begin{array}{l}\text { col. } \\
\text { sums }\end{array}$ & $\begin{array}{c}53 \\
(70 \%)\end{array}$ & $\begin{array}{c}23 \\
(30 \%)\end{array}$ & $\mathrm{N}=76$ & $\begin{array}{c}\text { col. } \\
\text { sums }\end{array}$ & $\begin{array}{c}69 \\
(73 \%)\end{array}$ & $\begin{array}{c}26 \\
(27 \%)\end{array}$ & $\mathrm{N}=95$ & $\begin{array}{l}\text { col. } \\
\text { sums }\end{array}$ & $\begin{array}{c}122 \\
(71 \%)\end{array}$ & $\begin{array}{c}49 \\
(29 \%)\end{array}$ & $\mathrm{N}=171$ \\
\hline
\end{tabular}

In a second replicate of the experiment (Dataset 2), we questioned $N=95$ fourth semester students (Table 1B). The corresponding odds ratio is 4.04; the one-sided Fisher's exact test results in $P=0.020$. In this replicate, students were 3.0 times more likely to observe a positive correlation if they expected to see one $(23 / 68=0.338$ vs. $3 / 27=0.111)$.

When we merged the two datasets for a joint analysis ( $\mathrm{N}=171$, Table $1 \mathrm{C})$, we found a combined odds ratio of 3.48. Overall, students were 2.6 times more likely to observe a positive correlation if they expected one $(P=0.0024 ; 42 / 119=0.353 \mathrm{vs.} 7 / 52=0.135)$. Thus, the two individual analyses as well as the joint analysis demonstrate that when analyzing a simple graph, students were strongly inclined towards observing what they expected to see.

\section{Controlling for competence in interpreting graphs}

The participants were not randomly assigned to the two groups of expectations; they assigned themselves based on their existing preconceived notions. It is thus possible that a significant difference in observations between the two groups could be attributed to a biased self-assignment. The discovery of Simpson's paradox and the corresponding positive in-age group correlation in Figure 1 is a non-trivial task of graphical data analysis; less than a third of students reported observing this (Table 1). If those students that are more proficient in the 
interpretation of graphically displayed data were biased towards expecting a positive correlation, then this could have confounded our analysis.

To control for this possibility, we asked the students in the second replicate of the experiment an additional third question. We displayed a graph with four panels of artificially created data displaying correlations of various strengths (Figure 2), asking students to order them from the strongest negative to the strongest positive correlation. We found that students that were able to correctly order the panels (b-d-a-c) did not expect a positive correlation more often than other students (odds ratio $0.76, P=0.63$ from two-sided Fisher's exact test in the combined dataset; Table 2). Thus, the group assignment was unbiased from the point of view of graphical data analysis competence.
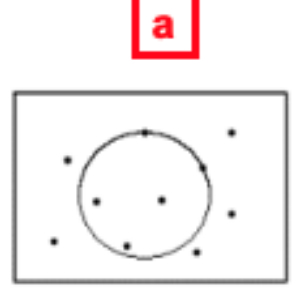

b

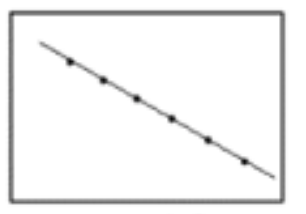

c

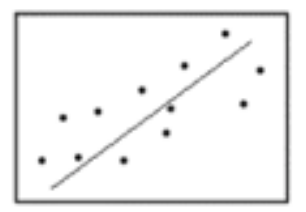

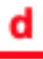

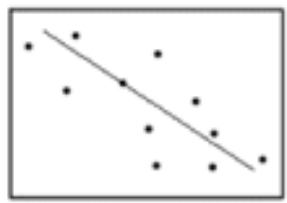

Figure 2. The panels of correlated data pairs displayed to the students for the control question.

Table 2. Contingency table contrasting the ability to correctly order the panels in Figure 2 with the group assignment according to the expected correlation (dataset 2).

\begin{tabular}{|c|c|c|c|}
\hline & $\begin{array}{c}\text { neg. } \\
\text { corr. } \\
\text { expect. }\end{array}$ & $\begin{array}{c}\text { pos. } \\
\text { corr. } \\
\text { expect. }\end{array}$ & $\begin{array}{c}\text { row } \\
\text { sums }\end{array}$ \\
\hline $\begin{array}{c}\text { incorrect } \\
\text { order }\end{array}$ & 8 & 26 & $\begin{array}{c}34 \\
(37 \%)\end{array}$ \\
\hline $\begin{array}{c}\text { orrect } \\
\text { order }\end{array}$ & 17 & 42 & $\begin{array}{c}59 \\
(63 \%)\end{array}$ \\
\hline col. sums & $\begin{array}{c}25 \\
(27 \%)\end{array}$ & $\begin{array}{c}68 \\
(73 \%)\end{array}$ & $\mathbf{N}=\mathbf{9 3}$ \\
\hline
\end{tabular}




\section{Discussion}

Our analysis shows that the two groups of participants - those expecting a negative and those expecting a positive correlation - showed a strong difference in how they interpreted a data plot. This experiment thus reveals an important aspect of the generation of interpretations from data. Although the act of drawing conclusions from a data plot appears to be a straightforward and transparent process, our results reveal that it is significantly guided by our expectations. While previous works have highlighted the large variation present in analysis workflows executed across research teams [1, 2], these studies have implicitly assumed that identical workflows would lead to the same interpretations. Here we contribute to this research with the surprising result that even the extremely isolated act of interpreting a plot can be strongly influenced by confirmation bias. This result suggests that each researcher reflects information uniquely through one's specific belief system, thus experiencing one's own reality.

A noteworthy limitation of our experiment is our ignorance of the exact thought process leading each participant to decide whether there is a positive or negative relationship. Further research is required to gain insight into precisely how confirmation bias influences the thought process of an individual interpreting a data plot. We speculate that a contradiction is present at the core of the plot interpretation: each age group on its own shows a positive correlation, while overall a negative correlation is observed (Figure 1). Resolving this instance of Simpson's paradox [14] required embracing the contradiction to understand how both aspects can be simultaneously true. In the face of such contradictions, participants may often fall back on their biases rather than focusing on the contradiction's resolution. In any scientific endeavour, contradictions should be a reason for joy, as they signal that the observer might have stumbled upon an interesting new aspect of the data.

That people interpret identical data plots in different ways according to preconceived notions has implications for how researchers should be trained in data analysis. One way to counter confirmation bias is a deliberate distinction between the 'day science' aspects of hypothesis testing and the 'night science' aspects of hypothesis generation [15-20]. In the latter, contradictions are embraced and pondered leading to the designs of new hypotheses and experiments. By enabling a culture where exploration is encouraged, the scientific community can better control for the confirmation bias present in each of our thought processes. Indeed, addressing these issues may help to mitigate the so-called 'reproducibility crisis' in science [21, 22].

\section{Methods}

In GNU R [R], we used linear functions with added Gaussian noise to create four subsets of data with positive correlations, the "age groups". These were then combined into a dataset with an overall negative correlation (Figure 1). 
Experiments were performed via the online conference software Cisco Webex ${ }^{\mathrm{TM}}$. After each slide, students were asked a question and wrote their answer as a personal message to the conductor of the experiment into the chat of the conference software. The anonymized responses of individual students are given in Suppl. Tables S1 (Dataset 1) and S2 (Dataset 2).

In each of the two replicate experiments, we first showed students an introductory slide that explained the premise of the dataset, stating that "A survey examines the connection between income and happiness. What relationship do you expect between these two parameters?" (the original slides with German text can be found in Suppl. File 1). In addition, the slide showed two simple plots with a clear positive and a clear negative correlation, respectively (see Suppl. File 1 , slide 1). Students were given 60 seconds to answer "p" for positive of " $n$ " for negative.

Next, the students were shown Figure 1 on a slide stating the following: "A survey examines the connection between income and happiness. Colors denote different age groups (1=youngest, $4=o l d e s t)$. Does the data suggest that a higher income leads to increased happiness?" Students were given 60 seconds to reply "y" for yes or " $n$ " for no.

In the second replicate of the experiment (Dataset 2), we added a third question. Students were shown the four panels in Figure 2, together with the following instruction: "Sort the four panels, from the strongest negative to the strongest positive correlation." Students were then given 100 seconds to write the panel order (e.g., "bdac") into the chat.

The data was aggregated from the chat history and analyzed in R [R]; the analysis script can be found in Suppl. File 2. As we hypothesized a positive relationship between expected and observed correlations, we used one-sided Fisher's exact tests for the analyses of the data in

Table 1. As we had no a priori expectation of a specific relationship between expected correlation and the proficiency in graphical data analysis tested by question 3 , we used a two-sided Fisher's exact test to analyse the data in Table 2.

\section{References}

1. Botvinik-Nezer R, Holzmeister F, Camerer CF, et al (2020) Variability in the analysis of a single neuroimaging dataset by many teams. Nature 582:84-88

2. Breznau N, Rinke EM, Wuttke A, et al (2021) Observing many researchers using the same data and hypothesis reveals a hidden universe of uncertainty. BITSS. https://doi.org/10.31222/osf.io/cd5j9

3. Del Giudice M, Gangestad SW (2021) A traveler's guide to the multiverse: Promises, pitfalls, and a framework for the evaluation of analytic decisions. Adv Methods Pract Psychol Sci 4:251524592095492

4. Landy JF, Jia ML, Ding IL, et al (2020) Crowdsourcing hypothesis tests: Making transparent how design choices shape research results. Psychol Bull 146:451-479

5. Simmons JP, Nelson LD, Simonsohn U (2011) False-positive psychology: undisclosed flexibility in data collection and analysis allows presenting anything as significant. Psychol 
Sci 22:1359-1366

6. Dutilh G, Annis J, Brown SD, et al (2019) The Quality of Response Time Data Inference: A Blinded, Collaborative Assessment of the Validity of Cognitive Models. Psychon Bull Rev 26:1051-1069

7. Bastiaansen JA, Kunkels YK, Blaauw FJ, et al (2020) Time to get personal? The impact of researchers choices on the selection of treatment targets using the experience sampling methodology. J Psychosom Res 137:110211

8. Huntington-Klein N, Arenas A, Beam E, et al (2021) The influence of hidden researcher decisions in applied microeconomics. Econ Inq. https://doi.org/10.1111/ecin.12992

9. Nickerson RS (1998) Confirmation bias: A ubiquitous phenomenon in many guises. Rev Gen Psychol 2:175-220

10. Pohl RF (2012) Cognitive Illusions: A Handbook on Fallacies and Biases in Thinking, Judgement and Memory. Psychology Press

11. Hergovich A, Schott R, Burger C (2010) Biased evaluation of abstracts depending on topic and conclusion: Further evidence of a confirmation bias within scientific psychology. Curr Psychol 29:188-209

12. Koehler JJ (1993) The influence of prior beliefs on scientific judgments of evidence quality. Organ Behav Hum Decis Process 56:28-55

13. Mahoney MJ (1977) Publication prejudices: An experimental study of confirmatory bias in the peer review system. Cognit Ther Res 1:161-175

14. Simpson EH (1951) The Interpretation of Interaction in Contingency Tables. Journal of the Royal Statistical Society: Series B (Methodological) 13:238-241

15. Yanai I, Lercher M (2019) Night science. Genome Biol 20:179

16. Yanai I, Lercher M (2019) What is the question? Genome Biol 20:289

17. Yanai I, Lercher M (2020) Renaissance minds in 21st century science. Genome Biol 21:67

18. Yanai I, Lercher M (2020) The two languages of science. Genome Biol 21:147

19. Yanai I, Lercher M (2020) A hypothesis is a liability. Genome Biol 21:231

20. Yanai I, Lercher M (2021) The data-hypothesis conversation. Genome Biol 22:58

21. Camerer CF, Dreber A, Holzmeister F, et al (2018) Evaluating the replicability of social science experiments in Nature and Science between 2010 and 2015. Nat Hum Behav 2:637-644

22. Ioannidis JPA (2005) Why most published research findings are false. PLoS Med 2:e124 


\section{Acknowledgements}

For their participation in the described experiment, we thank the students of the Introduction to Programming (winter semester 2020/2021) and the students of the Statistical Data Analysis course (summer semester 2021) at Heinrich Heine University Düsseldorf. 